\title{
High voltage and large current dynamic test of SiC diodes and hybrid module
}

\author{
Ao Liu ${ }^{1, a^{*}}$, Gang Chen ${ }^{1,2}$, Song Bai ${ }^{1,2}$, Run Hua Huang ${ }^{1}$, Yong hong Tao ${ }^{1}$, \\ Ling Wang ${ }^{1}$ \\ ${ }^{1}$ Nanjing Electronic Device Institute Nanjing, 210016, CHN \\ ${ }^{2}$ Science and Technology on Monolithic Integrated Circuits and Modules Laboratory \\ Nanjing, 210016, CHN \\ a E-mail: wsygdhra@126.com
}

Keywords: dynamic test; double-pulse; $\mathrm{SiC}$ diodes and modules.

Abstract. High voltage and large current dynamic test for $\mathrm{SiC}$ diodes and hybrid module was studied. Then high voltage dynamic test for 1700V-25A 4H-SiC junction barrier schottky (JBS) diodes and high current dynamic test for a 1700V-200A half-brige SiC hybrid module were performed. With the change of test conditions, such as driving resistance and load inductance, dynamic parameters have some variation. The reverse recovery time of SiC diode and swich on/off time of SiC hybrid module under test is between $20 \mathrm{~ns}$ to $200 \mathrm{~ns}$. The test got a high accuracy. These devices are very suitable for high frequency applications.

\section{Introduction}

With the development of power electronic device technology, Silicon Carbide ( $\mathrm{SiC}$ ) as a semiconductor material has superior wide band gap characteristics, which cannot be attained by conventional silicon ( $\mathrm{Si}$ ) semiconductors, thereby making them well suited for constructing switching devices in power electronic circuits ${ }^{[1]}$. In particular, smaller, more efficient equipment can be expected when a $\mathrm{SiC}$ device used in power conversion equipment ${ }^{[2]}$. Therefore, the authors are focusing on dynamic characteristics of $\mathrm{SiC}$ devices for operation under extremely high frequency.

Double pulse test method is usually used to test power electronic devices ${ }^{[3]}$. In this paper, dynamic test for 1700V-25A 4H-SiC junction barrier schottky (JBS) diodes and high current dynamic test for 1700V-200A half-bridge SiC hybrid module were performed. And some comparisons for different test conditions were discussed.

\section{Dynamic parameters and test methods}

The dynamic parameters of the diode are mainly reflected in the reverse recovery process. The main dynamic parameters include reverse recovery charge, reverse recovery current and reverse recovery time $\left(\mathrm{Q}_{\mathrm{rr}}, \mathrm{I}_{\mathrm{r}}, \mathrm{t}_{\mathrm{rr}}\right)$. Fig.1 shows the definition of these dynamic parameters. Reverse recovery charge is time integration of the reverse recovery current. Starting point of the integration is the moment when forward current of the diode is reduced to zero, finishing point of the integration is the moment when reverse recovery current reach $10 \%$ of the peak $\mathrm{I}_{\mathrm{Ir}}$.

For IGBT and MOSFET, Dynamic characteristics are divided into the the swich on process and the swich off process. In the swich-on process, The main dynamic parameters include each time interval $\left(t_{\text {don }}, t_{r}\right)$ and swich-on loss $\left(E_{o n}\right)$. Fig.2 shows each time interval $\left(t_{d o n}, t_{r}\right)$ and swich-on loss $\left(E_{\text {on }}\right)$ under inductive loads and specific conditions. Swich-on loss is the time integration of $\mathrm{V}_{\mathrm{CE}} \times \mathrm{I}_{\mathrm{C}}$. Starting point of the integration is the moment when the gate voltage reach $10 \% \mathrm{~V}_{\mathrm{GE}}$, finishing point of the integration is the moment when $\mathrm{V}_{\mathrm{CE}}$ is reduced to $2 \%$ of the peak $\mathrm{V}_{\mathrm{CE}}$.In the swich-off process, The main dynamic parameters include each time interval $\left(\mathrm{t}_{\text {doff }}, \mathrm{t}_{\mathrm{f}}\right)$ and swich-off $\operatorname{loss}\left(\mathrm{E}_{\mathrm{off}}\right)$. Fig.2 also shows each time interval $\left(t_{\text {doff }}, t_{f}\right)$ and swich off loss $\left(E_{\text {off }}\right)$ under inductive loads and specific conditions. Swich-off loss is the time integration of $\mathrm{V}_{\mathrm{CE}} \times \mathrm{I}_{\mathrm{C}}$. Starting point of the integration is the moment when 
the grid voltage reach $90 \% \mathrm{~V}_{\mathrm{GE}}$, finishing point of the integration is the moment when $\mathrm{I}_{\mathrm{C}}$ is reduced to $2 \%$ of the peak $\mathrm{I}_{\mathrm{C}}$.
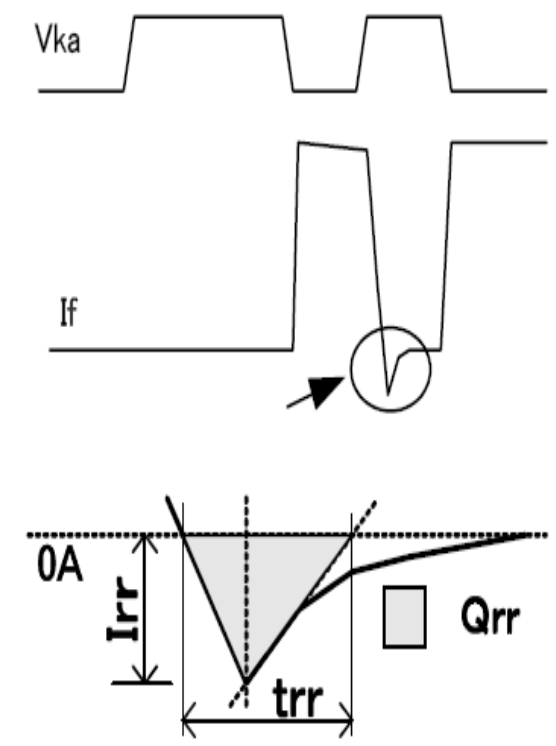

Fig.1 dynamic parameters of reverse recovery

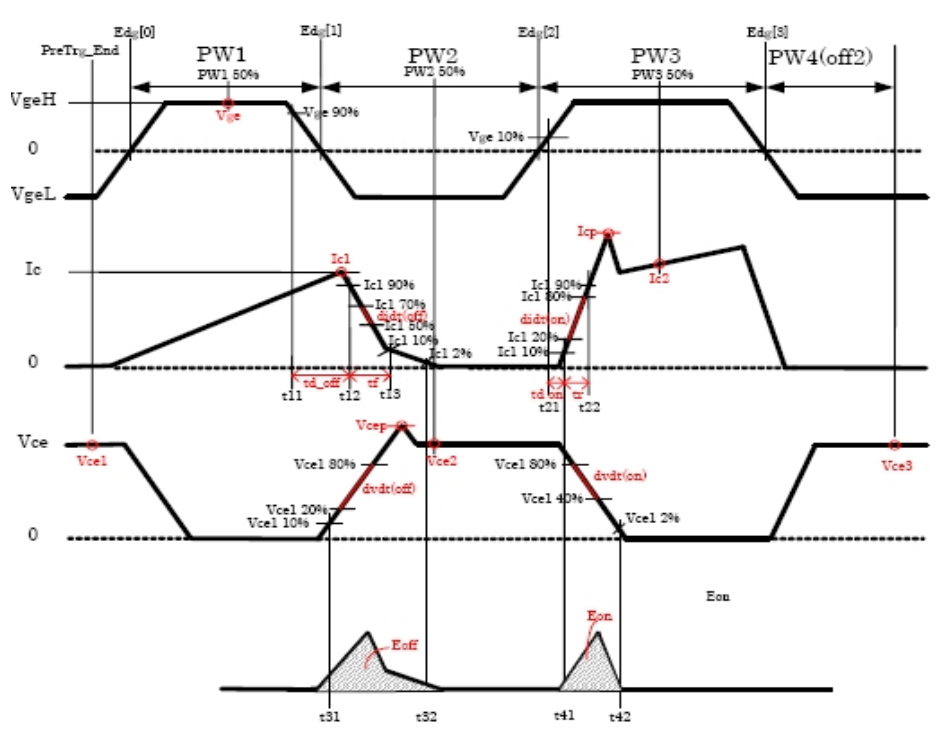

Fig.2 dynamic parameters of IGBTs

The dynamic parameters test mainly have two methods of single pulse and double pulse. Double pulse test method is used to test $\mathrm{SiC}$ device and hybrid module in this paper. Fig.3 shows principle diagram of double pulse test method. Lowside IGBT and highside diode are the devices under test. For $\mathrm{SiC}$ hybrid module, gate voltage of highside IGBT is negative .So, highside IGBT is in off-state, only free-wheeling diode is at work.

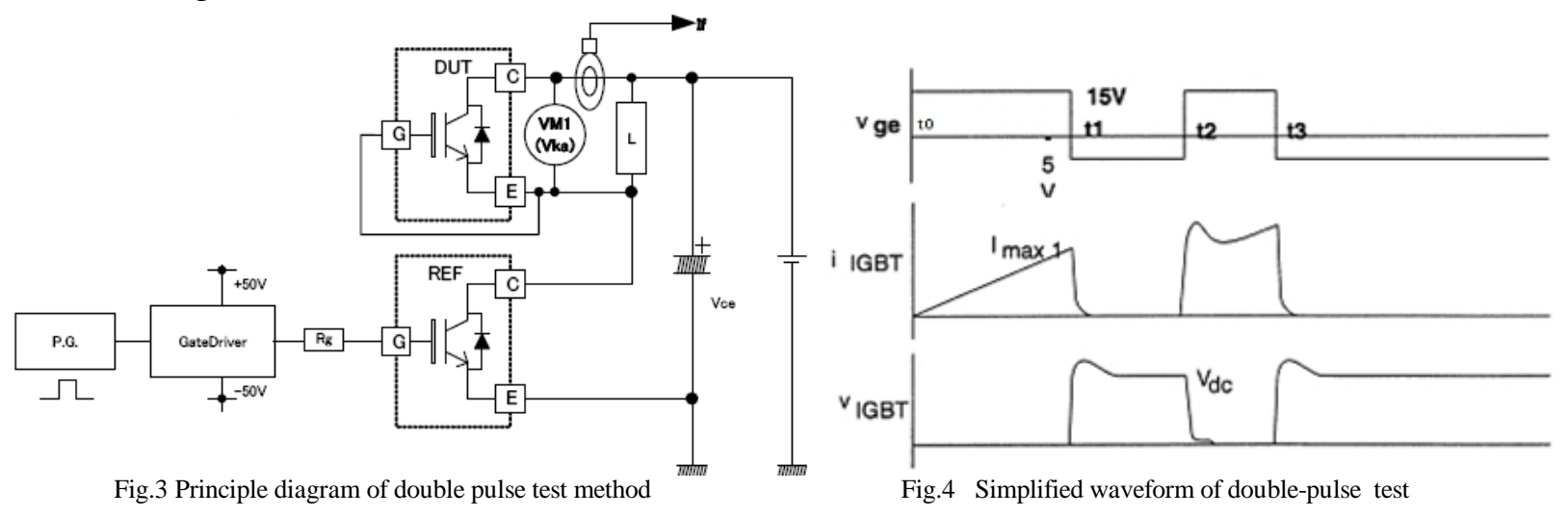

At the moment of $\mathrm{t}_{0}$, gatedriver gives first pulse to lowside IGBT . Lowside IGBT is at on-state, so the voltage $\mathrm{U}$ of DC power supply is applied to the inductive load L. Current I flowing through the inductance appears linear growth. Formula of the current is $\mathrm{I}=\mathrm{U} \times \mathrm{t} / \mathrm{L}$. At the moment of $\mathrm{t} 1$, the value of the current is determined by DC power supply and inductance. By adjusting the time $t_{1}$, specified current is get.

At the moment of $t_{1}$, lowside IGBT is turned off. The current on the inductance flow through highside free-wheeling diode. The current decays slowly. A current loop is constituted by inductance and highside diode.

At the moment of $\mathrm{t}_{2}$, gate driver gives second pulse to lowside IGBT, free-wheeling diode enter the reverse recovery process. At the same time, reverse recovery current flow into lowside IGBT.

At the moment of $t_{3}$, lowside IGBT is turned off again. Due to the stray inductance in the circuit and the capacitance of the device, a peak voltage may occur. Protection circuit should be designed to ensure the safety of the device. Fig.5 shows field test of high voltage dynamic parameters. 


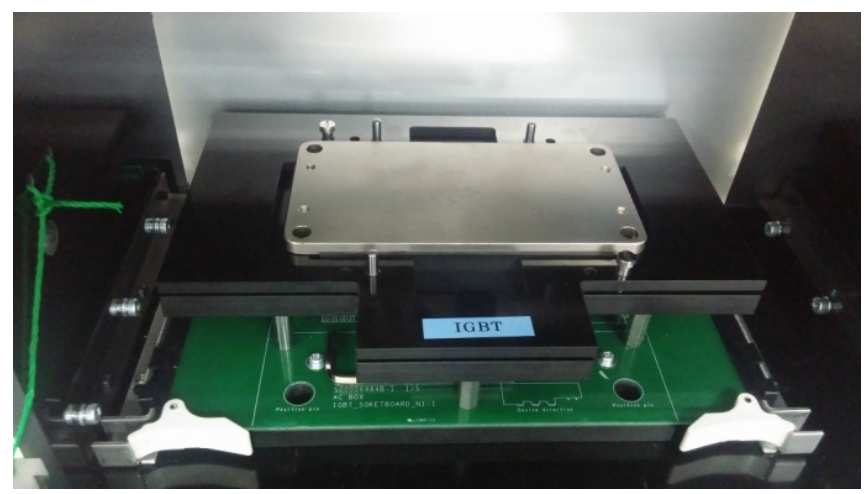

Fig.5 field test of high voltage dynamic parameters

\section{High voltage dynamic test}

1700V-25A 4H-SiC junction barrier schottky (JBS) diodes were designed and fabricated based on SiC epitaxy and device technology. The developed diodes have a short reverse recovery time at room temperature. These diodes have one-tenth lower recovery loss and one-third lower recovery time than those of a commercialized $1.7-\mathrm{kV} \mathrm{Si}$ diode $^{[4][5]}$. In order to give a more accurate value of reverse recovery parameters in such high voltage, this paper gives some comparisons under different test conditions.

DC power supply output is $1700 \mathrm{~V}$. SiC diode were tested under calculated conditions, which ensure the current flow through the diode is $25 \mathrm{~A}$ at the interval between two pulses. Fig. 6 shows the whole test process. Load inductance is selected to be $200 \mu \mathrm{H}$. In order to study the effect of driving resistance on reverse recovery, two sets of resistances were used to evaluate the reverse recovery process. First set, the driving resistance is $100 \Omega / 2 \Omega$ that respectively corresponds to lowside IGBT turn-on/ turn-off. Second set, the driving resistance chosen is $10 \Omega / 10 \Omega$.

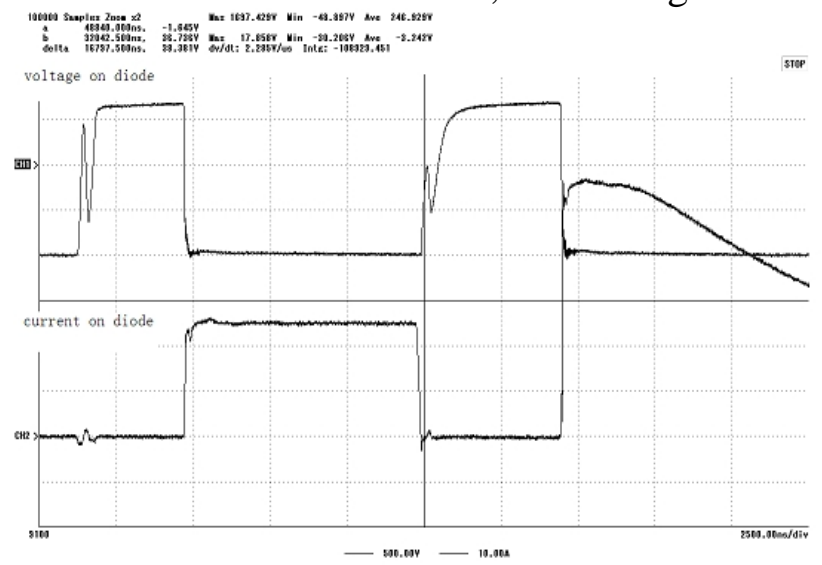

Fig.6 $1700 \mathrm{~V}$ and 25A reverse recovery process

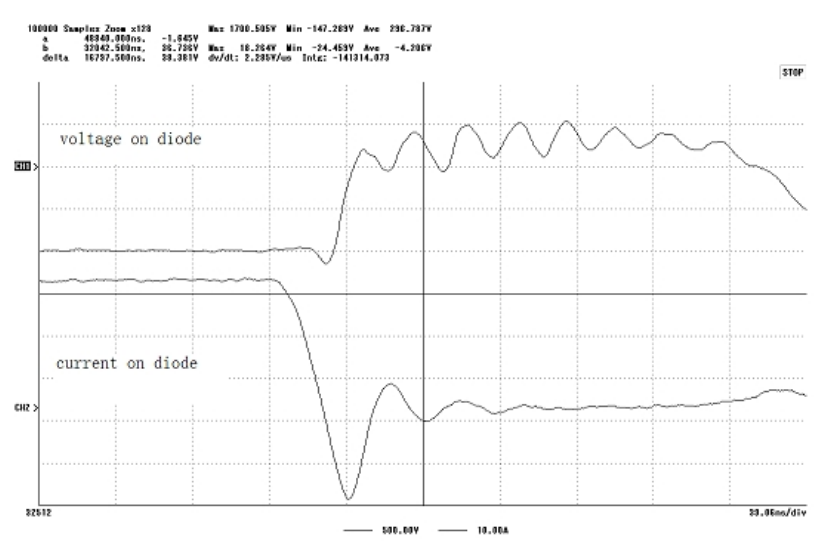

Fig. 8 reverse recovery process with driving resistance is $10 \Omega / 10 \Omega$

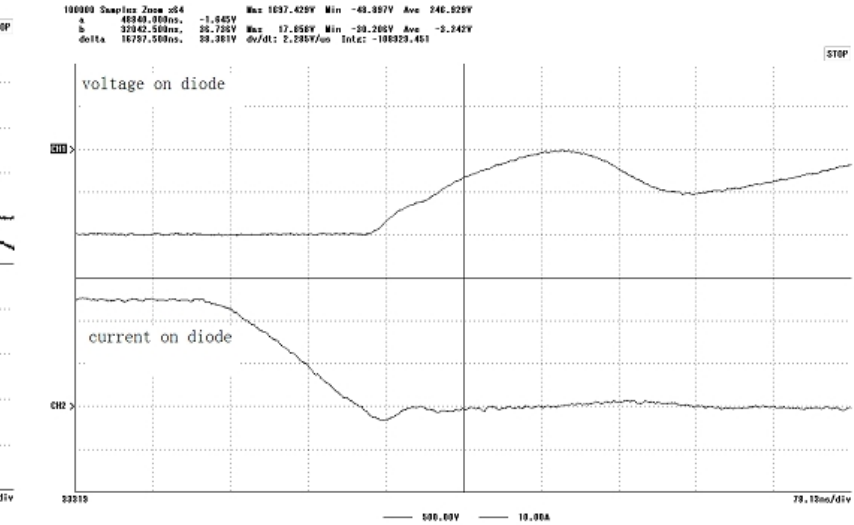

Fig.7 reverse recovery process with driving resistance is $100 \Omega / 2 \Omega$

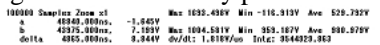

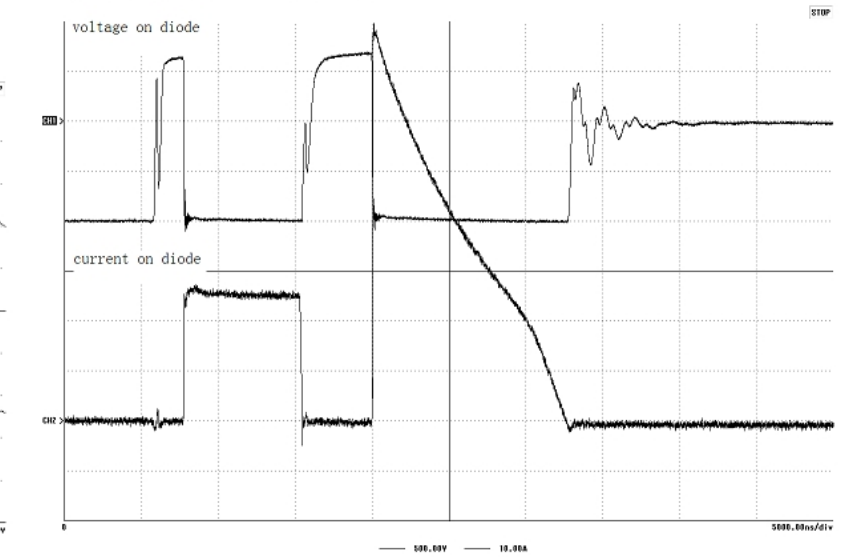

Fig.9 reverse recovery process with load inductance is $100 \mu \mathrm{H}$ 
At the condition that the driving resistance is $100 \Omega / 2 \Omega$,the speed of lowside IGBT turn-on is slow. So the rate of change of Current $(\mathrm{di} / \mathrm{dt})$ is $260 \mathrm{~A} / \mu \mathrm{s}$. Reverse recovery time is $52 \mathrm{~ns}$ and the peak of reverse recovery current is $3.4 \mathrm{~A}$. Thus, there is $3.1 \mathrm{~mJ}$ of reverse recovery loss on the diode, and reverse recovery charge is $99 \mathrm{nC}$.

In contrast, when the driving resistance is $10 \Omega / 10 \Omega$, the speed of lowside IGBT turn-on is much faster. SiC diode under tested gets a high rate of change of Current( di/dt) for $1902 \mathrm{~A} / \mu \mathrm{s}$. Reverse recovery time reduces to $24 \mathrm{~ns}$ and the peak of reverse recovery current increases to $21.7 \mathrm{~A}$. There is 4.4 $\mathrm{mJ}$ of reverse recovery loss on the diode, and reverse recovery charge is 330nC. In addition, the oscillation occurs which is triggered by not only the stray inductance of the package but also the device capacitance in the IGBT and the SiC diode. The choice of driving resistance is important in dynamic test.

A load inductance for $100 \mu \mathrm{H}$ is used in the test circuit to make a contrast test(Fig.9). The driving resistance is $100 \Omega / 2 \Omega$.Under smaller load inductance, reverse recovery dynamic parameters do not basically have a change. However, the current on the diode increase rapidly during the period of second pulses. For the safety of the device, a suitable load inductor needs to be selected.

\section{Large current dynamic test}

Large current dynamic test needs high power storage capacitor and cable. At the same time, parasitic parameters in the test circuit should be low enough. A 1700V-200A half-bridge SiC hybrid module was designed and fabricated as a sample to be tested. The Si IGBTs and SiC SBDs are attached on the direct bond copper substrate with minimum inductance loop. Eight 25A SiC SBDs are connected in parallel as one set of freewheeling diode. The solder joints both die and substrate, substrate and baseplate by the reflow solder systems.

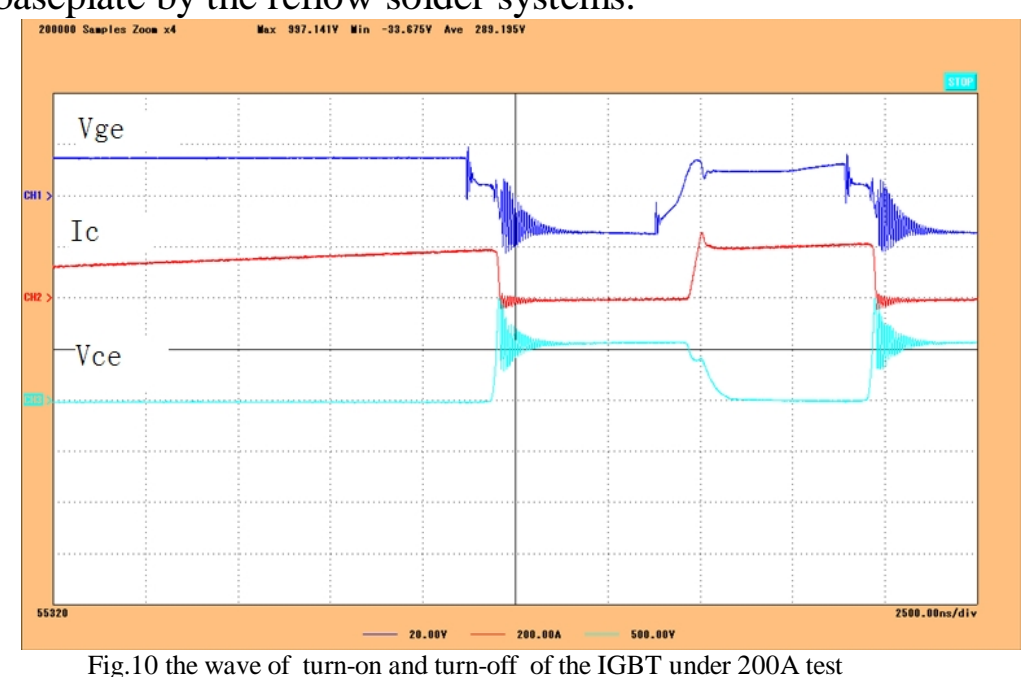




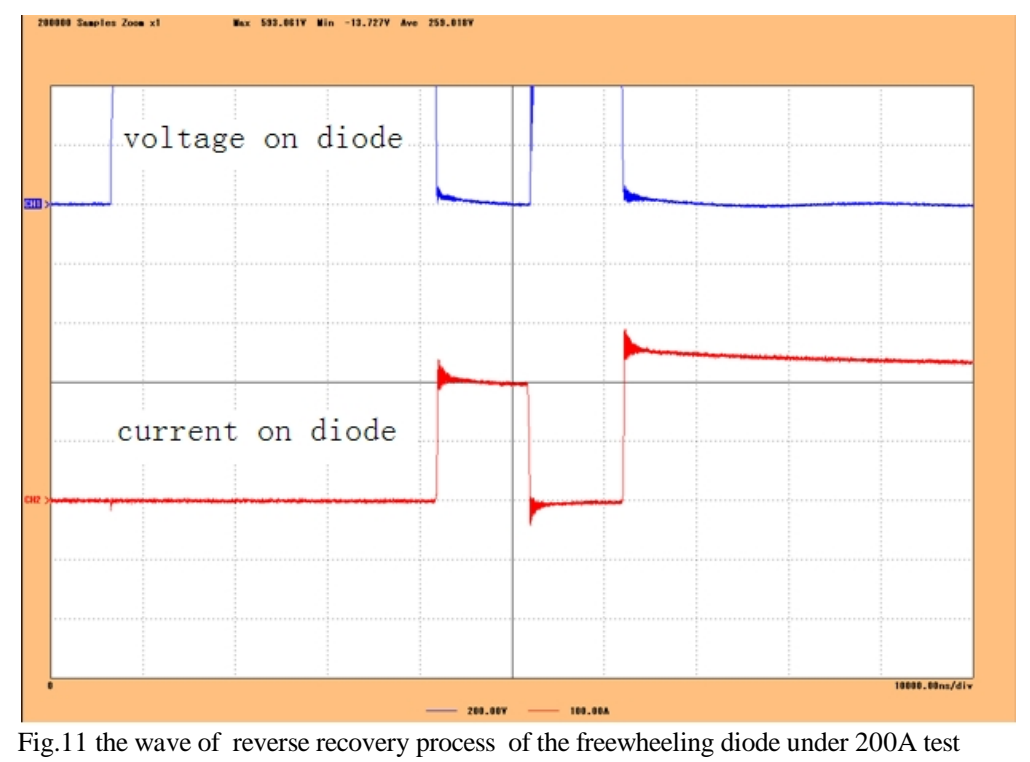

Dynamic test is under $600 \mathrm{~V}$, which is determined by the DC power supply. By a accurate calculation of the width of the pulse, turn-on and turn-off of the IGBTs is under the condition of 200A. The driving resistance is $20 \Omega / 20 \Omega$. In order to reach large current in short time, load inductance is selected to be $100 \mu \mathrm{H}$. Fig. 10 shows the wave of turn-on and turn-off of the IGBT . Delay time ton is 227ns. Rise time $t_{r}$ and fall time $t_{f}$ are 198ns and 68ns. By the integral operation, swich-on loss Eon and swich-off loss Eoff are $42 \mathrm{~mJ}$ and $12 \mathrm{~mJ}$.

Furthermore, reverse recovery process of freewheeling diode is tested under the same condition(Fig.11). Diodes under tested gets a high rate of change of Current( di/dt) for $600 \mathrm{~A} / \mu \mathrm{s}$. Reverse recovery time trr is $76 \mathrm{~ns}$ and reverse recovery charge is $560 \mathrm{nC}$. About $9 \mathrm{~mJ}$ of loss occurs during reverse recovery process.

\section{Summary}

This paper discusses the high voltage and large current dynamic parameters test for SiC diodes and hybird modules. Double-pulse test method was used to evaluate the device and module. This paper gives the definition of dynamic parameters and introduces the whole process of the test. A $1700 \mathrm{~V}-25 \mathrm{~A}$ 4H-SiC junction barrier schottky (JBS) diodes and A 1700V-200A half-brige SiC hybrid module were tested with the test method and circuit. Mainwhile, this paper discusses the influence of different test conditions, and find what are the main influencing factors. This paper gives the final dynamic parameters under different test conditions. The reverse recovery time of $\mathrm{SiC}$ diode and swich on/off time of $\mathrm{SiC}$ hybrid module under test is between 20ns to 200ns.

\section{References}

[1] Jovalusky J. New low reverse recovery charge (QRR) high voltage silicon diodes provide higher efficiency than presently available ultrafast rectifiers[C]. Proc. Twenty-Third Annual IEEE Applied Power Electronics Conference and Exposition APEC 2008, pp.918-923.

[2] Johnson C.M., Rahimo M., Wright, N.G., Hinchley, D.A., Horsfall A.B., Morrison, D.J., Knights A. Characterisation of $4 \mathrm{H}-\mathrm{SiC}$ Schottky diodes for IGBT applications[C]. Proc. IEEE Industry Applications Conference, 2000, Vol. 5, pp. 2941 - 2947.

[3] A.Elasser, M. Ghezzo. Switching characteristics of silicon carbide power PiN diodes[J]. Solid-State Electronics 44(2000) 317-323.

[4] Kang, I.H.;Kim, S.C. Accurate Extraction Method of Reverse Recovery Time and Stored Charge for Ultrafast Diodes[J]. IEEE Transactions on Power Electronics,2012. 27(2): 619-622. 
[5] Adamowicz, M. Giziewski, S. Performance comparison of SiC Schottky diodes and silicon ultra fast recovery diodes[C]. CPE 2011. 7th International Conference-Workshop , 2011, 144 - 149 